\title{
Particle-based non-Newtonian fluid animation for melting objects
}

\author{
Afonso Paiva, Fabiano Petronetto, Thomas Lewiner and Geovan Tavares \\ PUC-Rio - Department of Mathematics - Matmídia Laboratory — Rio de Janeiro — Brazil \\ \{apneto, fbipetro, tomlew, tavares\}@mat.puc-rio.br
}
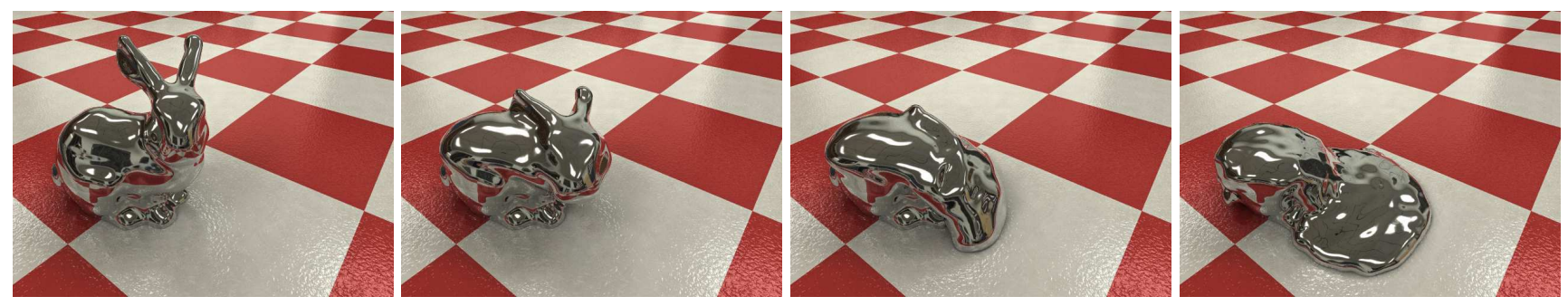

Figure 1. Melting the Stanford bunny starting cold at the bottom and hot at the top.

\begin{abstract}
This paper presents a new visually realistic animation technique for objects that melt and flow. It simulates viscoplastic properties of materials such as metal, plastic, wax, polymer and lava. The technique consists in modeling the object by the transition of a non-Newtonian fluid with high viscosity to a liquid of low viscosity. During the melting, the viscosity is formulated using the General Newtonian fluids model, whose properties depend on the local temperature. The phase transition is then driven by the heat equation. The fluid simulation framework uses a variation of the Lagrangian method called Smoothed Particle Hydrodynamics. This paper also includes several schemes that improve the efficiency and the numerical stability of the equations.
\end{abstract}

Keywords: Melting, Smoothed Particle Hydrodynamics, Non-Newtonian Fluid, Heat Equation, Physically Based Animation and Computational Fluid Dynamics.

\section{Introduction}

Physically based animations appeared in Computer Graphics for visually realistic computer animations. The main delicate point remains the discretisation the physical law applying on a real-world object. This involves finding an adequate formulation of the physics and stable approximation schemes for their discretisation. In this paper, we will focus on the heating of a solid viscoplastic object, such as the example of Figure 1. Parts of the object remains solid and parts becomes liquid, whose viscosity depends on its temperature. The heat equation will thus drive the phase transition.

Physical laws. Among non-Newtonian fluids, viscoplastic fluids are characterized by the toothpaste effect: a significant force must be applied to them before it starts to flow. The critical of the external force is known as the yield stress. During melting, the yield stress, and thus the viscosity, depends on the temperature. The recent advances of Mendes et al. [15] formulate the viscosity with a general Newtonian law which encompasses both viscous and liquid state. The conciseness and generality of this formulation suits better for melting, and we introduce it here for simulation.

Approximation scheme. Simulating the fluid behavior of a viscoplastic object in its liquid phase requires a computational fluid dynamics (CFD) framework. In the computer graphics literature, the most common CFD model relies on Eulerian formulation where physical quantities are sampled on a regular grid. This suits well for classical Newtonian fluids like water. However, controlling grid based methods require tracking the boundary of the fluid, which remains a laborious task in free flow simulations.

In this work, we use a Lagrangian formulation on a particle-based representation, called Smoothed Particle Hydrodynamics (SPH). The SPH method was introduced in 1977 by Gingold and Monaghan [7] and Lucy [13] simulate compressible fluids in astrophysics. Each particle rep- 
resents a small volume of fluid subjected to natural forces such as gravity, pressure and viscosity. SPH methods are simple to implement and its accuracy compares nicely to grid based methods in several instances.

Related works This work uses the SPH framework to simulate melting solid objects as a non-Newtonian fluid. We will quickly summarize the most relevant work to us on these three topics.

Smoothed Particle Hydrodynamics. The SPH method was introduced in the computer graphics community by Desbrun and Cani in [6], where they used SPH to simulate deformable bodies. Müller et al. [20] then used the SPH approach for simulating incompressible fluids with surface tension. Furthermore, they introduced point-splatting to capture the fluid free surface. Recently SPH methods became very popular in the special effects industry where it was used to simulate lava flow in the third part of The Lord of the Rings trilogy.

Non-Newtonian fluids. There are few works in computer graphics for non-Newtonian fluids. Goktekin et al. [8] proposed a grid-based method to compute the stress tensor of these fluids. They use a linear Maxwell model with von Mises plastic yield condition. Clavet et al. [3] use SPH with a linear combination of elastic springs between particles driven plastic yield condition. Another method using SPH was proposed by Mao and Yang [14], where the stress tensor derives from a corotational Maxwell model.

Melting. The idea of simulating melting and flowing of solids objects by coupling viscosity with temperature appeared in different contexts. Carlson et al. [2] use an Eulerian grid-based fluid method. They further need an implicit integrator to compensate the instability of the gridbased approach. Wei et al. [22] use cellular automata and replace Navier-Stokes equations by simple rules in each automata. Müller et al. [21] create a point-based framework to simulate elastoplastic objects with von Mises plastic yield condition. They use moving least squares (MLS) for approximating the velocity gradient. Finally, Keiser et al. in [10] introduces traditional SPH method in the previous framework with usual viscoplastic modeling.

Contributions. This paper introduces two new elements to the melting simulation. First, we use General Newtonian Fluid model [15] to compute the viscosity (see Section 2). Its concise formulation reduces the number of parameters for yield modeling and allows simpler relation with the temperature. Moreover, since this formulation covers viscoplastic fluids within a single equation, it treats the whole object at once and avoids delicate detection of the viscosity transition.

Moreover, we introduce in the heat equation approximation a more stable Laplacian operator on the particles (see Section 3). This approximation already improved Poisson simulations [5] by involving differences of first derivatives instead of second derivatives.

We also introduce several numerical improvements in the method. First, we introduce the use of XSPH [16] for avoiding the formation of stable clusters of particles (see Section 3). Then, we reintroduce the artificial viscosity [6], but for melting simulation. We also chose the continuity equation for density (equation (6)) to avoids particle outliers. Finally, our implementation uses an adaptive time step for each iteration based on the Courant-Friedrichs-Lewy condition (see Section 4).

\section{Formulation of the physical laws}

Computational fluid dynamics (CFD) aims at prediction fluid behavior through Navier-Stokes equations. These equations are commonly solved using conventional Eulerian formulation with grid-based methods such as finite differences and finite elements. In this work, we chose an alternative method driven by the Lagrangian formulation. As opposed to the Eulerian approach, the Lagrangian formulation does not require advective term, which suits well for meshless methods such as SPH. We will introduce now this formulation and the General Newtonian Fluid model [15] that models our viscoplastic object, together with our heating model. The reader will find further details on CFD in Anderson's book [1].

Lagrangian formulation. Navier-Stokes equations can be formulated by the following two equations describing the conservation of the mass (equation (1)) and of the momentum (equation (2)).

$$
\begin{gathered}
\frac{d \rho}{d t}=-\rho \nabla \cdot \mathbf{v} \\
\frac{d \mathbf{v}}{d t}=-\frac{1}{\rho} \nabla p+\frac{1}{\rho} \nabla \cdot \mathbf{S}+\mathbf{g}
\end{gathered}
$$

where $t$ denotes the time, $\mathbf{v}$ the velocity vector, $\rho$ the density, $p$ the pressure, $\mathbf{g}$ the gravity acceleration vector and $\mathbf{S}$ the viscoplastic stress tensor. This last term plays a fundamental role in melting simulation.

Generalized Newtonian Fluid model. For nonNewtonian fluids, the stress tensor is a nonlinear function of the deformation tensor $\mathbf{D}=\nabla \mathbf{v}+(\nabla \mathbf{v})^{T}$. For our simulation, we will use the Generalized Newtonian Liquid model proposed by Mendes et al. [15], where the stress 


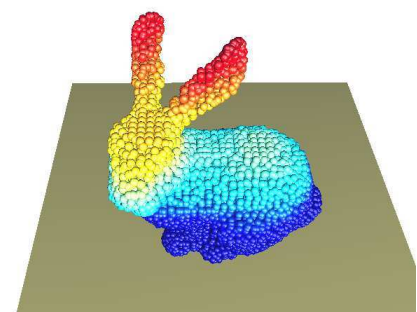

(a) Initial temperature.

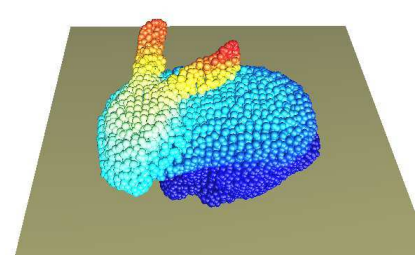

(b) 500 iterations.

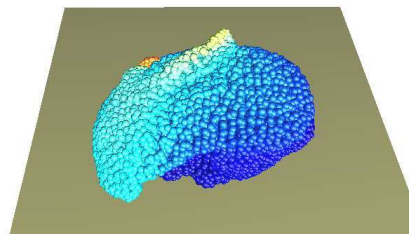

(c) 1060 iterations

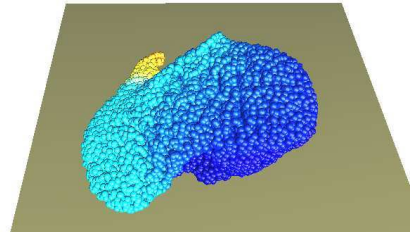

(d) 3000 iterations

Figure 2. Temperature of the 9727 particles of the bunny of Figure 1: the dark blue parts are below the melting point, and thus remain solid. Observe that the left ear of the bunny gets colder after touching the body.

tensor $\mathbf{S}$ is given by $\mathbf{S}=\eta(D) \mathbf{D}$, where the apparent viscosity $\eta$ depends on the intensity of deformation $D=\sqrt{\frac{1}{2} \cdot \operatorname{trace}(\mathbf{D})^{2}}$. The viscosity function $\eta$ is then given by:

$$
\eta(D)=(1-\exp [-(J+1) D])\left(D^{n-1}+\frac{1}{D}\right)
$$

where $n$ is the behavior of power-law index and $J$ is the jump number.

The jump number $J$ is a new rheological parameter of a viscoplastic fluid which combines previous ones such as the yield stress and the consistency index. In our simulation, we fixed $n=\frac{1}{2}$, and let only the jump number $J$ vary with the temperature.

Heating and melting. The melting of volumetric objects corresponds to a phase transition from solid to fluid. We can model this transition by varying the viscosity according to the temperature of each particle. This model was used in other animation frameworks, either using grid-based approach [2] or particle-based approach [10].

The time variation of the temperature is described by the following heat equation, involving the temperature $T$ and the thermal diffusion constant $k$ :

$$
\frac{d T}{d t}=k \nabla^{2} T
$$

When the temperature of some part of the object increases and reaches the melting point, it becomes liquid (see Figure 2). The jump number $J$ then decreases according to the temperature. We will model the jump number as decreasing linearly with respect to the temperature:

$$
J(T)=(1-u) J_{\max }-u J_{\min }
$$

with $u=\left(T-T_{\min }\right) /\left(T_{\max }-T_{\min }\right)$. Note that the viscosity function of equation (3) thus decreases when temperature increases and vice-versa.

\section{Particle-based approximation scheme}

We will use here the Smoothed Particle Hydrodynamics (SPH) framework for simulating the fluid behavior. In our animation framework, we use the formulation of SPH for incompressible fluid [17]. A wide review of SPH methods can be found in Monaghan's survey [18].

The SPH principles are reviewed in Section 3.1. It requires a discretisation of the different terms of the governing equations: Section 3.2 describes the approximation of the density for the continuity equation (1), Section 3.3 describes the approximation of the pressure and the stress tensor of the momentum equation (2). Sections 3.4 and 3.5 present corrections for the viscosity and the velocity. Finally, Section 3.6 introduces our approximation of the Laplacian in the heat equation (4).

\subsection{Smoothed Particle Hydrodynamics}

The key idea of SPH is to replace the fluid by a set of particles (see Figure 3). The dynamics of the fluid is then naturally governed by the Lagrangian version of the NavierStokes equations introduced in the last Section. The local fluid properties such as mass and volume are attached to each particle and interpolated in-between particles. This interpolation uses a smoothing kernel $W$ on the particles in a radius of $h$. A scalar field $A(\mathbf{x})$ and its associated gradient vector field $\nabla A(\mathbf{x})$ at point $\mathbf{x}$ are interpolated using the particles $j$ within a disk of radius $h$ around $\mathbf{x}$ as follows:

$$
\begin{gathered}
A(\mathbf{x})=\sum_{j=i}^{n} A\left(\mathbf{x}_{j}\right) \frac{m_{j}}{\rho_{j}} W\left(\mathbf{x}-\mathbf{x}_{j}, h\right) \\
\nabla A(\mathbf{x})=\sum_{j=i}^{n} A\left(\mathbf{x}_{j}\right) \frac{m_{j}}{\rho_{j}} \nabla W\left(\mathbf{x}-\mathbf{x}_{j}, h\right)
\end{gathered}
$$

where $n$ is the number of neighboring particles, $j$ the particle index, $\mathbf{x}_{j}$ the particle position, $m_{j}$ the particle mass and $\rho_{j}$ the particle density. 

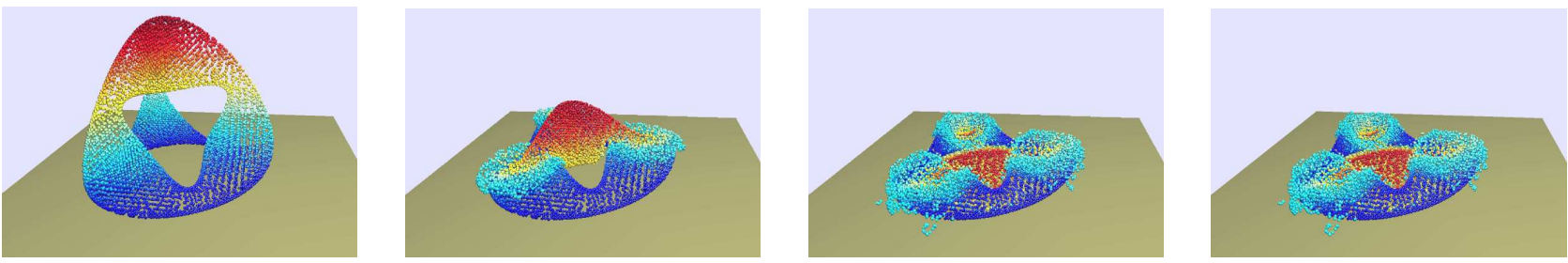

Figure 3. SPH schemes deal gracefully with complex topological of the chair surface.

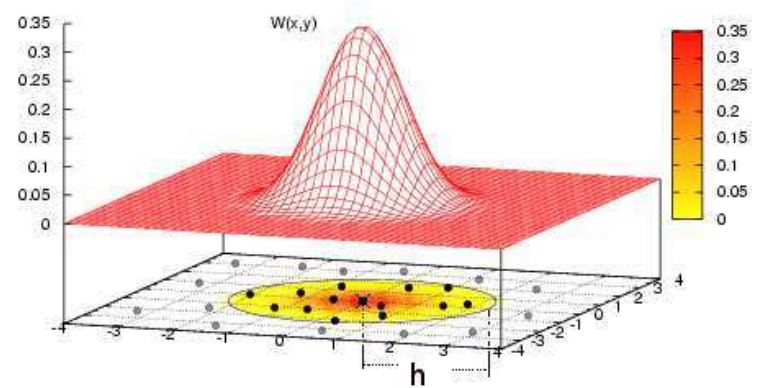

fluid interface, which leads to spurious results. Moreover, it requires more computational efforts since the density must be evaluated before other parameters, such as pressure. We therefore chose another approximation for the density, using the following SPH version of continuity equation (1):

$$
\frac{d \rho_{i}}{d t}=\rho_{i} \sum_{j=1}^{n} \frac{m_{j}}{\rho_{j}}\left(\mathbf{v}_{i}-\mathbf{v}_{j}\right) \cdot \nabla_{i} W\left(\mathbf{x}_{i j}, h\right) .
$$

where $\mathbf{v}_{i}$ and $\mathbf{v}_{j}$ are velocities at particles $i$ and $j$ respectively, and $\mathbf{x}_{i j}=\mathbf{x}_{i}-\mathbf{x}_{j}$.

\subsection{Particle approximation of the momentum}

Figure 4. Quintic smoothing kernel: the particles farther than the smoothing length $h$ are not considered in the convolution.

In this work, we choose a piecewise quintic smoothing kernel function (see Figure 4),

$$
\begin{gathered}
W\left(\mathbf{x}-\mathbf{x}_{j}, h\right)=\frac{3}{359} \pi h^{3} \cdot w\left(\frac{\left\|\mathbf{x}-\mathbf{x}_{j}\right\|}{h}\right), \text { with: } \\
w(q)=\left\{\begin{array}{r}
(3-q)^{5}-6(2-q)^{5}+15(1-q)^{5} ; 0 \leq q<1 \\
(3-q)^{5}-6(2-q)^{5} ; 1 \leq q<2 \\
(3-q)^{5} ; 2 \leq q \leq 3 \\
0 ; \quad q>3
\end{array}\right.
\end{gathered}
$$

\subsection{Particle approximation of continuity}

Density is usually approximate in SPH systems using the density summation, which follows directly from the $\mathrm{SPH}$ approximation of equation (5):

$$
\rho_{i}=\sum_{j=i}^{n} m_{j} W\left(\mathbf{x}_{i}-\mathbf{x}_{j}, h\right)
$$

However, to ensure the physical meaning of the approximation, we need to introduce a symmetrization between the pressure and the local velocity [12]. In particular, the density summation approach has a particle deficiency near the

Pressure. The modeling of pressure remains a delicate point for SPH simulations of incompressible fluids, due to the lack of explicit control of the local density. Since SPH suits better for compressible fluid, we approximate the incompressible fluid by a quasi-compressible fluid through an equation of state [17] for the pressure. We use the one proposed by Morris et al. [19]:

$$
p_{i}=c^{2}\left(\rho_{i}-\rho_{0}\right)
$$

where $p_{i}$ is the pressure at particle $i, c$ the speed of sound, which represents the fastest velocity of a wave propagation in that medium, and $\rho_{0}$ is a reference density. This equation of state is very similar with the ideal gas equation of state used by Desbrun and Cani [6].

After updating the pressure at all particles using equation (7), we can evaluate the pressure term in equation (2) at each particle $i$ using a symmetrization similar to the density case [12]:

$$
-\frac{1}{\rho_{i}} \nabla p_{i}=-\sum_{j=1}^{n} m_{j}\left(\frac{p_{i}}{\rho_{i}^{2}}+\frac{p_{j}}{\rho_{j}^{2}}\right) \nabla_{i} W\left(\mathbf{x}_{i j}, h\right) .
$$

Stress tensor. In order to compute the stress tensor $\mathbf{S}_{i}=$ $\eta\left(D_{i}\right) \mathbf{D}_{i}$ at each particle $i$, where $\eta\left(D_{i}\right)$ is given by the equation (3), we must pre-compute the deformation tensor:

$$
\mathbf{D}_{i}=\nabla \mathbf{v}_{i}+\left(\nabla \mathbf{v}_{i}\right)^{T}
$$




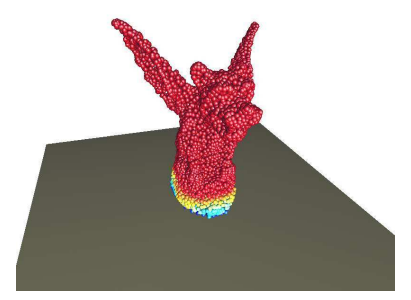

(a) 20 iterations.

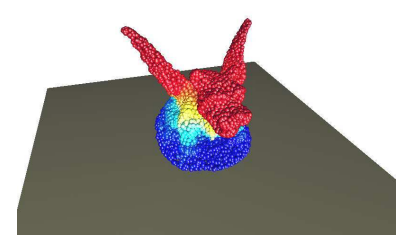

(b) 550 iterations

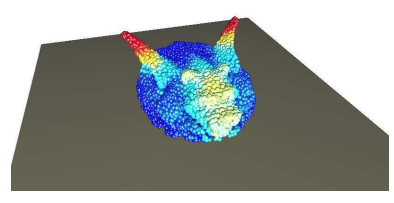

(c) 780 iterations.

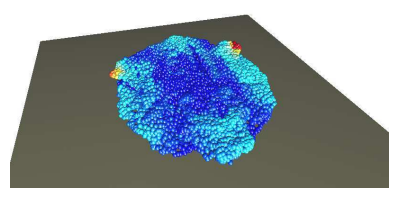

(d) 1320 iterations

Figure 5. Melting a completely liquid Gargoyle model using 6976 particles: the color codes the velocity of each particle. The stability of the method preserves the shape of the object without explicit mesh representation even after many iterations.

where the velocity is evaluated by the following equation:

$$
\nabla \mathbf{v}_{i}=\sum_{j=1}^{n} \frac{m_{j}}{\rho_{j}}\left(\mathbf{v}_{j}-\mathbf{v}_{i}\right) \otimes \nabla_{i} W\left(\mathbf{x}_{i j}, h\right) .
$$

Finally, after updating of the stress tensor $\mathbf{S}_{i}$ at each particle $i$, the stress term in equation (2) can be approximated by:

$$
\frac{1}{\rho_{i}} \nabla \cdot \mathbf{S}_{\mathbf{i}}=\sum_{j=1}^{n} \frac{m_{j}}{\rho_{i} \rho_{j}}\left(\mathbf{S}_{i}+\mathbf{S}_{j}\right) \cdot \nabla_{i} W\left(\mathbf{x}_{i j}, h\right) .
$$

\subsection{Viscosity correction}

To avoid numerical instabilities due to oscillations in the velocity vector field, which may ruin the simulation, a common technique adds an artificial viscous stress term in the $\mathrm{SPH}$ approximation of the linear momentum (equation (2)) as follows:

$$
\frac{d \mathbf{v}_{i}}{d t} \leftarrow \frac{d \mathbf{v}_{i}}{d t}-\sum_{j=1}^{n} \frac{m_{j}}{\rho_{i}} \prod_{i j} \nabla_{i} W\left(\mathbf{x}_{i j}, h\right) .
$$

The effect of the artificial viscous stress is given by the term:

$$
\prod_{i j}=\left\{\begin{array}{cl}
\frac{-\alpha \mu_{i j} c+\beta \mu_{i j}^{2}}{0.5\left(\rho_{i}+\rho_{j}\right)}, & \left(\mathbf{v}_{i}-\mathbf{v}_{j}\right) \cdot\left(\mathbf{x}_{i}-\mathbf{x}_{j}\right)<0 \\
0, & \left(\mathbf{v}_{i}-\mathbf{v}_{j}\right) \cdot\left(\mathbf{x}_{i}-\mathbf{x}_{j}\right) \geq 0
\end{array}\right.
$$

and

$$
\mu_{i j}=\frac{h\left(\mathbf{v}_{i}-\mathbf{v}_{j}\right) \cdot\left(\mathbf{x}_{i}-\mathbf{x}_{j}\right)}{\left|\mathbf{x}_{i}-\mathbf{x}_{j}\right|^{2}+0.01 h^{2}}
$$

where $\alpha$ corresponds to bulk viscosity and $\beta$ corresponds to von Neumann-Ritchmyer viscosity [18].

\subsection{XSPH Velocity correction}

To prevent particle inter-penetration, which may result in stable clusters of particles, Monaghan [16] introduced an improvement called XSPH velocity-correction. In XSPH ( $\mathrm{X}$ means unknown), each particle $i$ moves in the following way:

$$
\mathbf{v}_{i} \leftarrow \mathbf{v}_{i}+\varepsilon \sum_{j=1}^{n} \frac{m_{j}}{0.5\left(\rho_{i}+\rho_{j}\right)}\left(\mathbf{v}_{j}-\mathbf{v}_{i}\right) W\left(\mathbf{x}_{i j}, h\right)
$$

where $\varepsilon \in[0,1]$.

The XSPH technique consists in computing an average velocity from the velocities of the neighboring particles, it helps to keep particles of an incompressible flow to move more orderly.

\subsection{Laplacian approximation}

The Heat equation (4), which governs the phase transition from solid to fluid, requires an approximation for the Laplacian of the temperature $\nabla^{2} T_{i}$. This second derivative can be approximated using the normal SPH convolution by the use of second derivatives for each particle $i$ [10]:

$$
\nabla^{2} T_{i}=\sum_{j=1}^{n} \frac{m_{j}}{\rho_{j}}\left(T_{i}-T_{j}\right) \nabla_{i}^{2} W\left(\mathbf{x}_{i j}, h\right) .
$$

However, the above equation has some disadvantages such as sensibility to particle disorder: the heat transfer between particles may be positive or negative since the second derivatives can change sign. The heat equation using this approximation may not conserve the thermal energy in the adiabatic enclosure [4].

For these reasons, we use a Laplacian operator involving only first derivatives, which was first proposed by Cummins and Rudman [5] to solve the Poisson equation with the SPH version of the Projection Method. Its expression follows:

$$
\nabla^{2} T_{I}=\sum_{j=1}^{n} \frac{m_{j}}{\rho_{j}}\left(\frac{4 \rho_{i}}{\rho_{i}+\rho_{j}}\right)\left(T_{i}-T_{j}\right) \frac{\mathbf{x}_{i j} \cdot \nabla_{i} W\left(\mathbf{x}_{i j}, h\right)}{\left|\mathbf{x}_{i j}\right|^{2}+0.01 h^{2}} .
$$




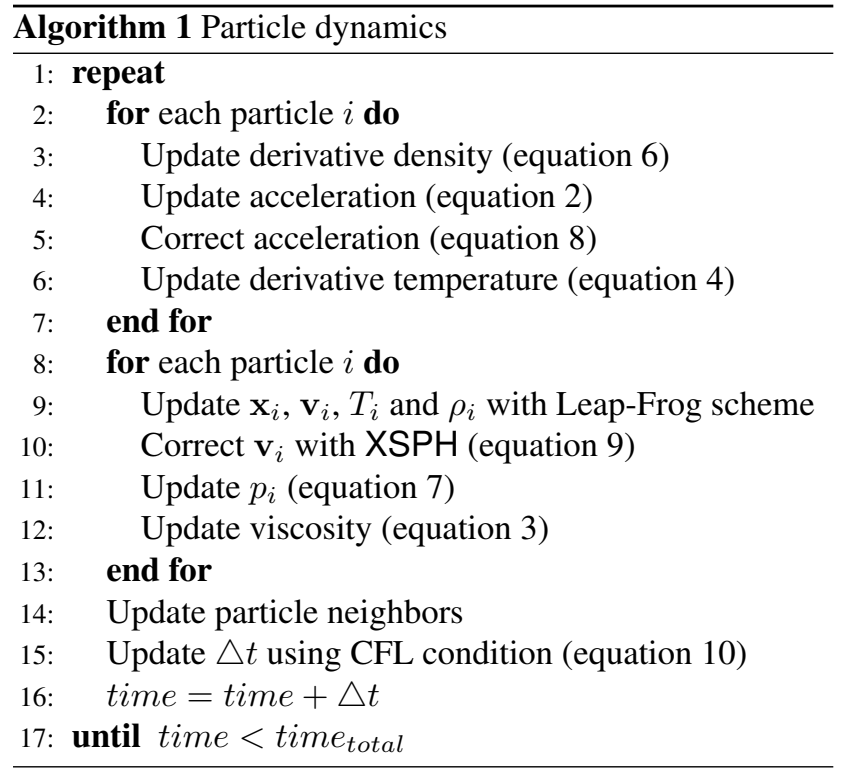

\begin{tabular}{lc} 
Attribute & Description \\
\hline \hline $\mathbf{x}$ & position \\
$\mathbf{v}$ & velocity \\
$\mathbf{a}$ & acceleration \\
$\mathbf{D}$ & deformation tensor \\
$\rho$ & density \\
$\eta$ & viscosity \\
$T$ & temperature \\
\end{tabular}

Table 1. Particle attributes.

\section{Results and Implementation}

In implementing a particle system we have two descriptions a global one and local one. The local description takes care of a single particle entity and of the attribute stored at each particle. In our model there are system attributes and particle attributes. The particle system attributes like mass, speed of sound and the smoothing length $h$ are global and they do not change with respect to time. The particle attributes vary with respect to time thus they must be stored at each particle, these attributes are given by table 1 . These attributes are updated in the sequence of algorithm 1.

\subsection{Numerical integration}

The SPH fluid equations are integrated with the second order accurate Leap-Frog scheme [12]. The advantages of Leap-Frog algorithm are computational efficiency for one fluid equation evaluation per step and the low memory stor- age required in the evaluation. The stability in this explicit time integration scheme is due to the Courant-FriedrichsLewy (CFL) condition, where adaptive time step is given by

$$
\triangle t=0.1 \min \left\{\frac{h}{\left|\mathbf{v}_{\max }\right|+c^{2}}, \frac{h^{2}}{6 \eta_{\max }}\right\} .
$$

This adaptive time step allows reducing the number of iteration while maintaining accuracy (see Figure 6).

\subsection{Neighbors retrieval}

In contrast with grid-base methods, where the positions of neighboring grid-cells are well defined, the neighbors of a given particle in the SPH method can vary with time. An adaptive hierarchy tree search [9] is adopted to find the particle neighbors.

The tree search method splits recursively the problem domain into octants that contain particles, until the leaves on the tree has a maximum particle number (we use at most ten particles for each leaf). After the tree structure is built, the search process can be performed.

For a given particle $i$, a cube with side $6 h$ centered at $\mathbf{x}_{i}$ is used to enclose the particle. We check at each level of the tree if the cube intersects the tree nodes containing the particles. If they do not intersect we stop the descent down on that particular path. If they do intersect we go to the next level and repeat the process until we reach the tree leaves containing particles. Now we check if each particle is inside of the support domain of the current particle $i$. If it is we record it as a particle neighbor. The complexity of this tree search method is of order $\mathcal{O}(n \log (n)), n$ being the total particle number.

\subsection{Rendering}

The tracking of the fluid free surface is done by rendering an isosurface from the SPH approximation of its characteristic function:

$$
\chi(\mathbf{x})=\sum_{j=1}^{n} \frac{m_{j}}{\rho_{j}} W\left(\mathbf{x}-\mathbf{x}_{j}, h\right)
$$

where the isovalue is in the range $[0,1]$.

We use an efficient and robust implementation of the marching cubes algorithm [11] to generate the triangle mesh for the isosurface. To improve the evaluation at the grid nodes we use the same hierarchical data structure for search neighboring particles.

The animations of Figures 1 and 6 were rendered using the open-source ray tracer POV-Ray. 


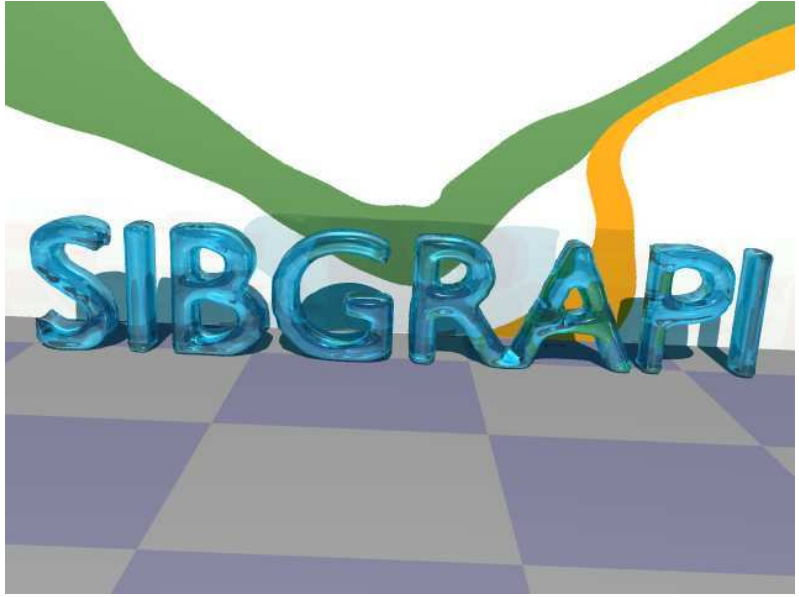

(a) Initial object.

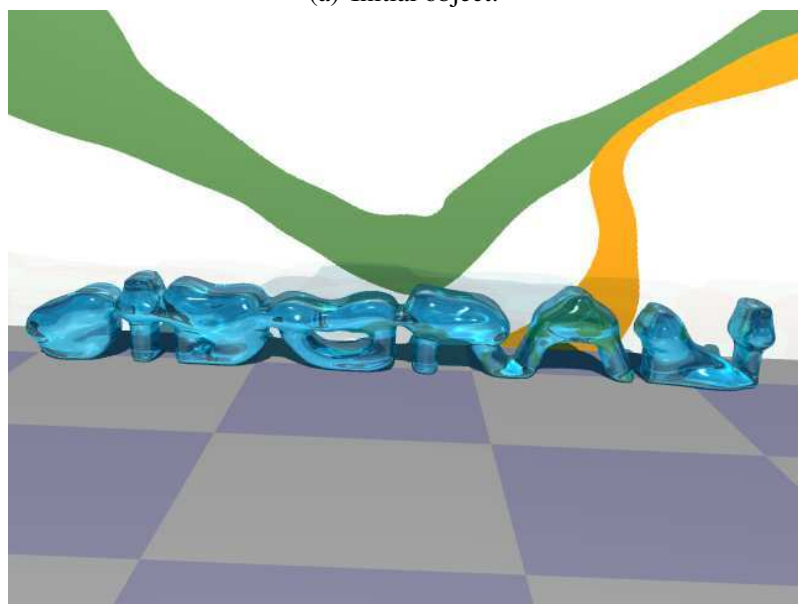

(c) 800 iterations.

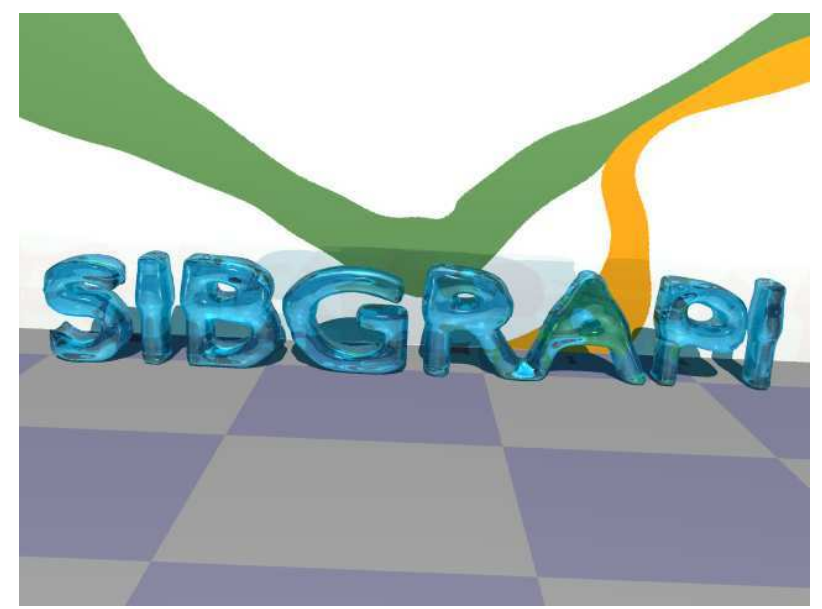

(b) 390 iterations.

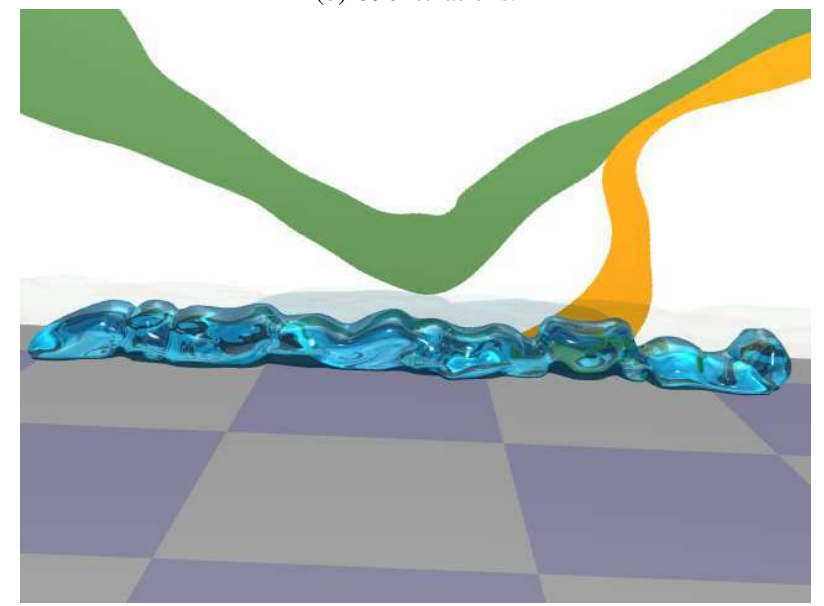

(d) 2000 iterations.

Figure 6. Melting of the SIBGRAPI logo using 12900 particles, starting cold at the bottom and hot at the top. The adaptive time step allows an accurate simulation with few iterations.

\subsection{Results}

We tested the method described in this paper on simple models. The example of Figures 1 and 2 simulates the melting of the Stanford bunny with 9727 particles. The simulation is initialized with a linear gradient of temperature such that the ears melt while the body remains cold and solid. The visual result of Figure 1 matches the intuition of the process. Moreover, the physical behavior is coherent, especially since one of the ears gets colder when touching the body, while the other one remains hot (see Figure 2).

In this work, we combined many advanced discretisation schemes to guarantee the stability of the simulation. The SPHmethod already offers simple handling of topological singularities, as for the 10000 particles simulation of Figure 3. This efforts result impressively when simulating the only flowing part of the melting, as on Figure 5. In that case, all the 6976 particles start above the melting point, and flows as a non-Newtonian fluid. The good handling of viscosity in SPH techniques allows very realistic results. For example, the head of the gargoyle remains well defined even when almost completely melted.

Finally, the proposed adaptive time step allows efficient simulations. For example, the melting of the Sibgrapi logo of Figure 6 used 12900 particles, i.e. more particles than for the bunny. However, it required less execution time for the simulation (see Table 2). This is due to the lower density of the model, which allowed bigger time steps.

\section{Conclusions and future works}

This paper proposed a physical simulation for melting viscoplastic objects. Our simulation relies on the SPH framework, and implements the General Newtonian Fluid 
model for viscoplastic fluids. It is further enhanced in numerical stability by adapted discretisation of the NavierStokes equation terms, and by a stable Laplacian operator for the heat equation. The effectiveness of the method is illustrated on simple examples which match the physical laws, leading to an efficient scheme for both animation and simulation purposes.

This work can be improved mainly in two directions. On one side, the inner nature of SPH systems permits a straightforward parallelization of the algorithm, which would increase the possible number of particles used during the simulation. On the other side, the rendering remains a fundamental part for animation purposes. The isosurfacing approach may be complemented by advanced rendering techniques during the simulation, in order to produce the final animation directly.

\begin{tabular}{ccc}
\hline Animation & $\begin{array}{c}\text { Number of } \\
\text { particles }\end{array}$ & $\begin{array}{c}\text { Time per } \\
\text { iteration }\end{array}$ \\
\hline \hline Bunny & 9727 & $0.94 \mathrm{~s}$ \\
Chair & 10000 & $0.75 \mathrm{~s}$ \\
Gargoyle & 6976 & $1.06 \mathrm{~s}$ \\
Sibgrapi & 12900 & $0.92 \mathrm{~s}$
\end{tabular}

Table 2. Average timings of the example animations running on Pentium $4-2.4 \mathrm{GHz}$. Note that in the Gargoyle simulation, all particles were fluids.

\section{Acknowledgments}

We would like to thank Prof. Paulo Roberto Mendes (Department of Mechanical Engineering, PUC-Rio) for suggesting us to use his viscoplastic fluid model. The authors are members of Matmidia laboratory at PUC-Rio which is sponsored by CNPq, FAPERJ and Petrobras.

\section{References}

[1] J. D. Anderson. Computational Fluid Dynamics. McGrawHill, 1995.

[2] M. Carlson, P. Mucha, B. Van Horn III, and G. Turk. Melting and flowing. In Proceedings of ACM SIGGRAPH Symposium on Computer Animation, pages 167-174, 2002.

[3] S. Clavet, P. Beaudoin, and P. Poulin. Particle-based viscoelastic simulation. Proceedings of $A C M S I G$ GRAPH/Eurographics Symposium on Computer Animation, pages 219-228, 2005.
[4] P. W. Cleary and J. J. Monaghan. Conduction modelling using smoothed particle hydrodynamics. Journal of Computational Physics, 148:227-264, 1999.

[5] S. J. Cummins and M. Rudman. An sph projection method. Journal of Computational Physics, 152:584-607, 1999.

[6] M. Desbrun and M. P. Cani. Smoothed particles: A new paradigm for animating highly deformable bodies. In Computer Animation and Simulation '96, pages 61-76. Procedings of EG Workshop on Animation and Simulation, SpringerVerlag, August 1996.

[7] R. A. Gingold and J. J. Monaghan. Smoothed particle hydrodynamics: theory and application to non-spherical stars. Monthly Notices of the Royal Astronomical Society, 181:375-389, 1977.

[8] T. G. Goktekin, A. W. Bargteil, and J. F. O'Brien. A method for animating viscoelastic. ACM Transactions on Graphics, 23(3):463-468, 2004.

[9] L. Hernquist and N. Katz. Treesph: A unification of sph with hierarchical tree method. The Astrophysical Journal of Supplement Series, 70:419-446, 1989.

[10] R. Keiser, B. Adams, D. Gasser, P. Bazzi, P. Dutré, and M. Gross. A unified lagrangian approach to solid-fluid animation. In Proceedings of the Eurographics Symposium on Point-Based Graphics, pages 125-134, 2005.

[11] T. Lewiner, H. Lopes, A. W. Vieira, and G. Tavares. Efficient implementation of marching cubes with topological guarantees. Journal of Graphics Tools, 8(2):234-241, 2003.

[12] S. Li and W. K. Liu. Meshfree Particle Methods. Springer, 2004.

[13] L. B. Lucy. Numerical approach to testing the fission hyphotesis. Astronomical Journal, 82:1013-1024, 1977.

[14] H. Mao and Y. Yang. A particle-based model for nonnewtonian fluid. Technical Report TR05-05, University of Alberta, 2005.

[15] P. R. S. Mendes, E. S. S. Dutra, J. R. R. Siffert, and M. F. Naccache. Gas displacement of viscoplastic liquids in cappilary tubes. Journal of Non-Newtonian Fluid Mechanics, 2005. (to appear).

[16] J. J. Monaghan. On the problem of penetration in particle methods. Journal of Computational Physics, 82:1-15, 1989.

[17] J. J. Monaghan. Simulating free surface flow with sph. Journal of Computational Physics, 110:399-406, 1994.

[18] J. J. Monaghan. Smoothed particle hydrodynamics. Reports on Progress in Physics, 68:1703-1759, 2005.

[19] J. P. Morris, P. J. Fox, and Y. Zhu. Modeling low reynolds number for incompressible flows using sph. Journal of Computational Physics, 136:214-226, 1997.

[20] M. Müller, D. Charypar, and M. Gross. Particle-based fluid simulation for interactive applications. In Proceedings of ACM SIGGRAPH/Eurographics Symposium on Computer Animation, pages 154-159, 2003.

[21] M. Muller, R. Keisser, A. Nealen, M. Pauly, M. Gross, and M. Alexa. Point based animation of elastic, plastic and melting. Proceedings of ACM SIGGRAPH/Eurographics Symposium on Computer Animation, pages 141-151, 2004.

[22] X. Wei, W. Li, and A. Kaufman. Melting and flowing of viscous volumes. Computer Animation and Social Agents (CASA), pages 54-59, 2003. 